\title{
Post-kala-azar Dermal Leishmaniasis Associated With AIDS*
}

\author{
Achiléa Bittencourt, Nancy Silva, Andréa Straatmann, \\ Victor Luiz Correia Nunes, Ivonise Follador and Roberto Badaró
}

\author{
Universitary Hospital Prof. Edgard Santos, Federal \\ University of Bahia; Roberto Santos Hospital of the \\ State of Bahia, Salvador, BA, Brazil
}

\begin{abstract}
Post-kala-azar dermal leishmaniasis (PKDL) is rarely reported in South America. In spite of the fact that there are many reports about the association of visceral leishmaniasis and AIDS, PKDL is very uncommon in HIV-positive patients, and so far only four cases have been documented in the literature. We present another case with unusual clinicopathological aspects. The patient, a 28-year-old male, from Salvador, Bahia (an endemic area) presented with clinical manifestations of visceral leishmaniasis three years after the diagnosis of AIDS. During treatment for visceral leishmaniasis he developed disseminated miliary papules. Microscopically, the skin biopsy showed a "saw-tooth" pattern with a lichenoid mononuclear infiltrate simulating lichen planus. The histopathological diagnosis was achieved through the finding of amastigotes. The authors discuss the clinicopathological aspects of this case based on a review of the specific literature.

Key Words: Visceral leishmaniasis, post-kala-azar dermal leishmaniasis, AIDS.
\end{abstract}

Post-kala-azar dermal leishmaniasis (PKDL) is frequent in India and the Sudan. In India PKDL occurs in 6-20\% of the cases of visceral leishmaniasis (VL), six months to five years after apparently successful treatment of VL [1]. In Africa PKDL is a common complication of $\mathrm{VL}$, occurring in $57 \%$ of the cases. However, a significantly higher PKDL rate (69\%) is observed in those patients who received inadequate or irregular treatment. African PKDL appears during or shortly after treatment [2].

PKDL manifests as multiple hypochromic macules, erythematous papules and nodules [2,3]. Usually, an erythematous macular eruption on the cheeks is the first manifestation of the disease [3]. A papular or nodular rash is the most frequent clinical aspect observed in $51 \%$ of the cases [2].

Received on 18 August 2002; revised 30 November 2002.

Address for correspondence: Dr. Achiléa L Bittencourt, Serviço de Anatomia Patológica, Hospital Universitário Prof. Edgard Santos, Rua João da Botas s/n, Zip code: 40110-060, E-mail: achilea@uol.com.br.*This paper should replace the manuscript published in volume 6, number 6, pages 313-316, with the same title.

The Brazilian Journal of Infectious Diseases 2002;7(3):229-233 (c) 2003 by The Brazilian Journal of Infectious Diseases and Contexto Publishing. All rights reserved. $1413-8670$
In spite of the fact that there are many reports about the association of VL and AIDS [4-8], PKDL is very uncommon in HIV-positive patients, and so far only four cases have been documented in the literature [912]. In these cases the lesions consisted of macules, papulo-nodular rash or erythematous plaques. The lesions were localized or involved only a few areas of the body. No case of micropapular rash has been observed.

In the South American literature PKDL is rarely reported $[13,14]$. We present a case of PKDL associated with AIDS with unusual clinicopathological aspects.

\section{Case Report}

A 28-year-old male, Negro, from the peripheral and coastal area of Salvador, Bahia, (endemic zone for visceral leishmaniasis) has had AIDS since 1997, when he first presented fever, diarrhea, convulsive crises, and loss of weight. Since then, he has regularly used an anti-retroviral therapy schedule with D4T, DDI, and nelfinavir, with disappearance of the symptomatology. Three years after the diagnosis of AIDS, he presented fever, loss of weight and hepatosplenomegaly. At this 
time he had a diagnosis of VL, due to a positive serological test for leishmaniasis and the presence of amastigotes in the bone marrow aspirate. He underwent treatment with Glucantime $(5525 \mathrm{mg} /$ day during 30 days), with great improvement. Two weeks after hospital discharge, before beginning the maintenance treatment, he developed a skin rash on the face, thorax, and on the upper and lower limbs. At this time he also presented hepatosplenomegaly and the direct examination and culture of one skin lesion was positive for leishmania.

\section{Dermatological examination}

A rash of multiple and confluent, miliary papules were seen over the face, on the anterior and posterior aspects of the thorax (Figures 1 and 2) and on the lower and upper limbs.

\section{$\underline{\text { Pathological examination }}$}

Histologic examination of a cutaneous biopsy specimen showed hyperkeratosis, a "saw-tooth" pattern, basal layer vacuolization, pigmentary incontinence and a lichenoid infiltration in the upper dermis (Figure 3). The inflammatory infiltrate was composed of plasma cells, lymphocytes, and macrophages, most of them containing amastigotes (Figure 4). In the lower dermis there was a similar perivascular infiltration.

\section{Discussion}

VL may present an acute, subacute or chronic evolution, but most infected individuals remain completely asymptomatic [15]. However association with conditions that cause immunosuppression can lead to progression from an asymptomatic form to the classic disease [16]. The patient lived in an area endemic for VL and probably had the asymptomatic form of VL before becoming infected with HIV.

The clinical pattern of the present case resembles keratosis pilaris, lichen spinulosus, phrynoderma, or miliaria rubra. It is similar to the micropapular form of PKDL previously described [2].
Histologically, in PKDL, a mild to heavy inflammatory infiltrate of plasma cells, lymphocytes, macrophages, parasitized or not, and occasional epithelioid and giant cells are seen. In cases with heavy infiltration an epidermal atrophy is observed $[2,14]$. According to Zijlstra et al. (2000) [2], in Sudan, only $20 \%$ of the skin biopsies show parasitism. In India, Ramesh and Mukherjee (1995) [3] found parasites in $58 \%$ of the biopsies. When there is association with AIDS amastigotes are frequent in the cutaneous lesions [9-12], as we observed here. Because of the great frequency of parasites PKDL may simulate borderline or subpolar diffuse leishmaniasis [17], however the clinical history and the clinical evaluation of the present case allowed a differential diagnosis. Histologically the lesion exhibited a "saw-tooth" pattern, a basal layer vacuolization and a lichenoid infiltrate, that could be misdiagnosed as lichen planus if the parasites were not observed.

This case demonstrates that it is mandatory to consider the diagnosis of PKDL in disseminated papulary skin lesions occurring during or after the treatment of VL.

\section{Acknowledgment}

Dr. Roque Almeida for the culture of the lesion.

\section{References}

1. Singh N., Ramesh V., Arora V.K., et al. Nodular post-kalaazar dermal leishmaniasis: a distinct histopathological entity. J Cutan Pathol 1998;25:95-9.

2. Zijlstra E.E., Khalil E.A.G., Kager P.A., et al. Post-kalaazar dermal leishmaniasis in the Sudan: clinical presentation and differential diagnosis. Brit J Dermatol 2000;143:136-43.

3. Ramesh V., Mukherjee A. Post-kala-azar dermal leishmaniasis. Int J Dermatol 1995;34:85-91.

4. Gramiccia M., Gradoni L., Troiani M. HIV-Leishmania co-infections in Italy. Isoenzyme characterization of Leishmania causing visceral leishmaniasis in HIV patients. Trans Royal Soc Trop Med Hig 1992;86:161-3.

5. Peters B.S., Fish D., Golden R., et al. Visceral leishmaniasis in HIV infection and AIDS: Clinical features and response to therapy Quart J Med 1990;77:1101-11. 
Figure 1. Military and confluent papules disseminated throughout the posterior aspect of the thorax.

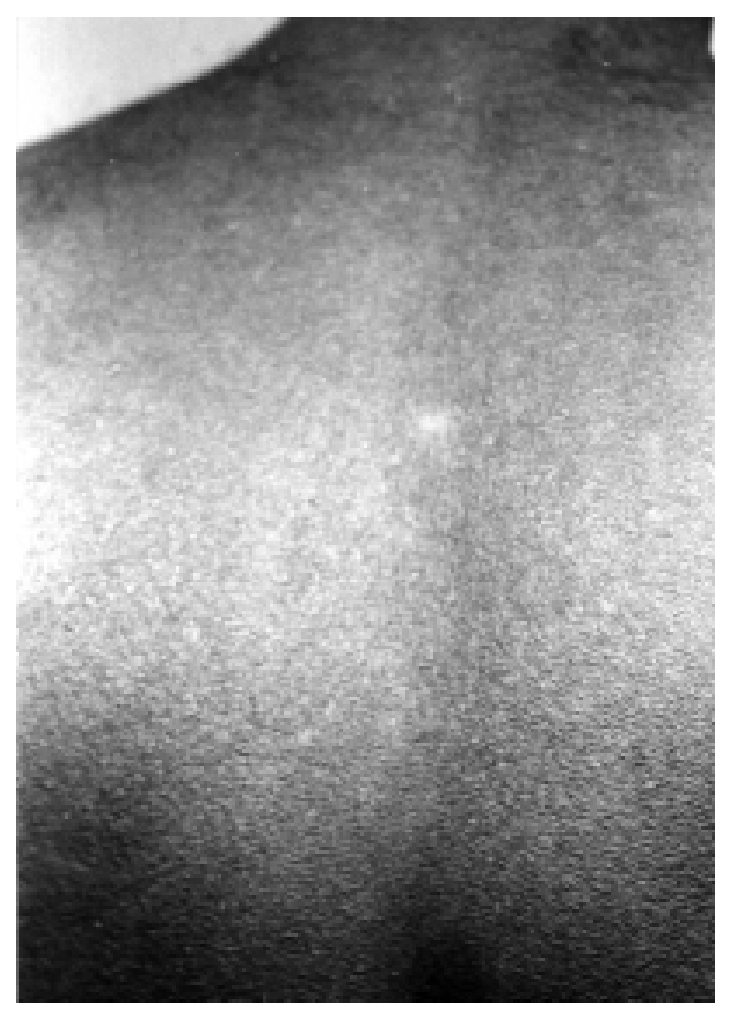

Figure 2. Numerous hyperkeratotic and miliary papules on the anterior aspect of the thorax.

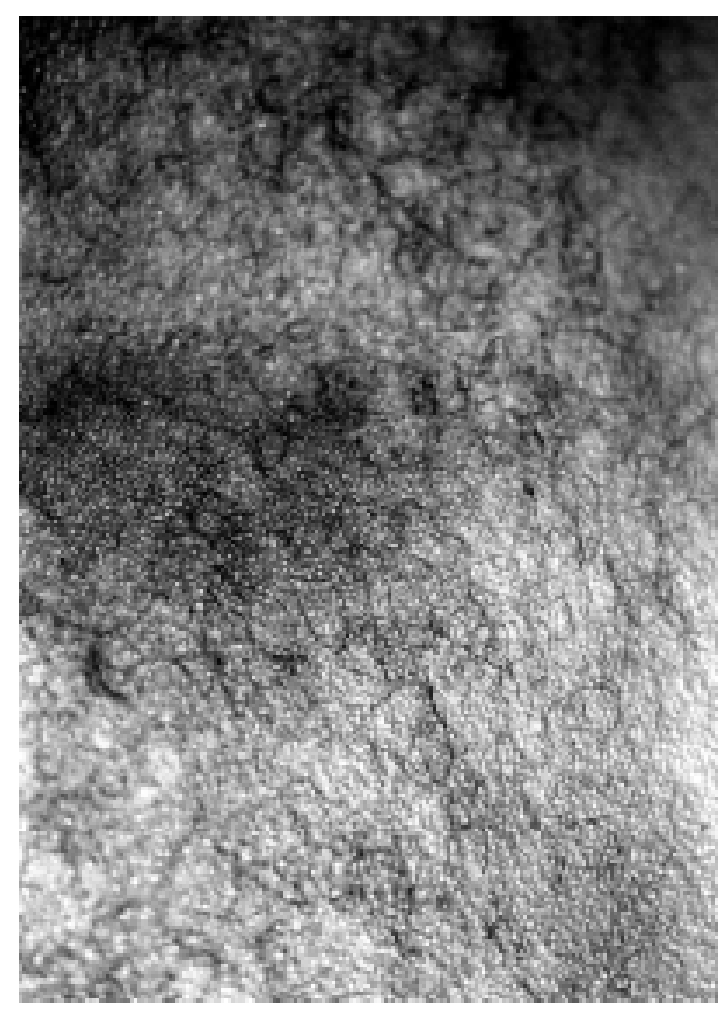


Figure 3. One entire papule. See a "saw-tooth" acanthosis and a lichenoid infiltrate in the upper dermis. HE, A40.

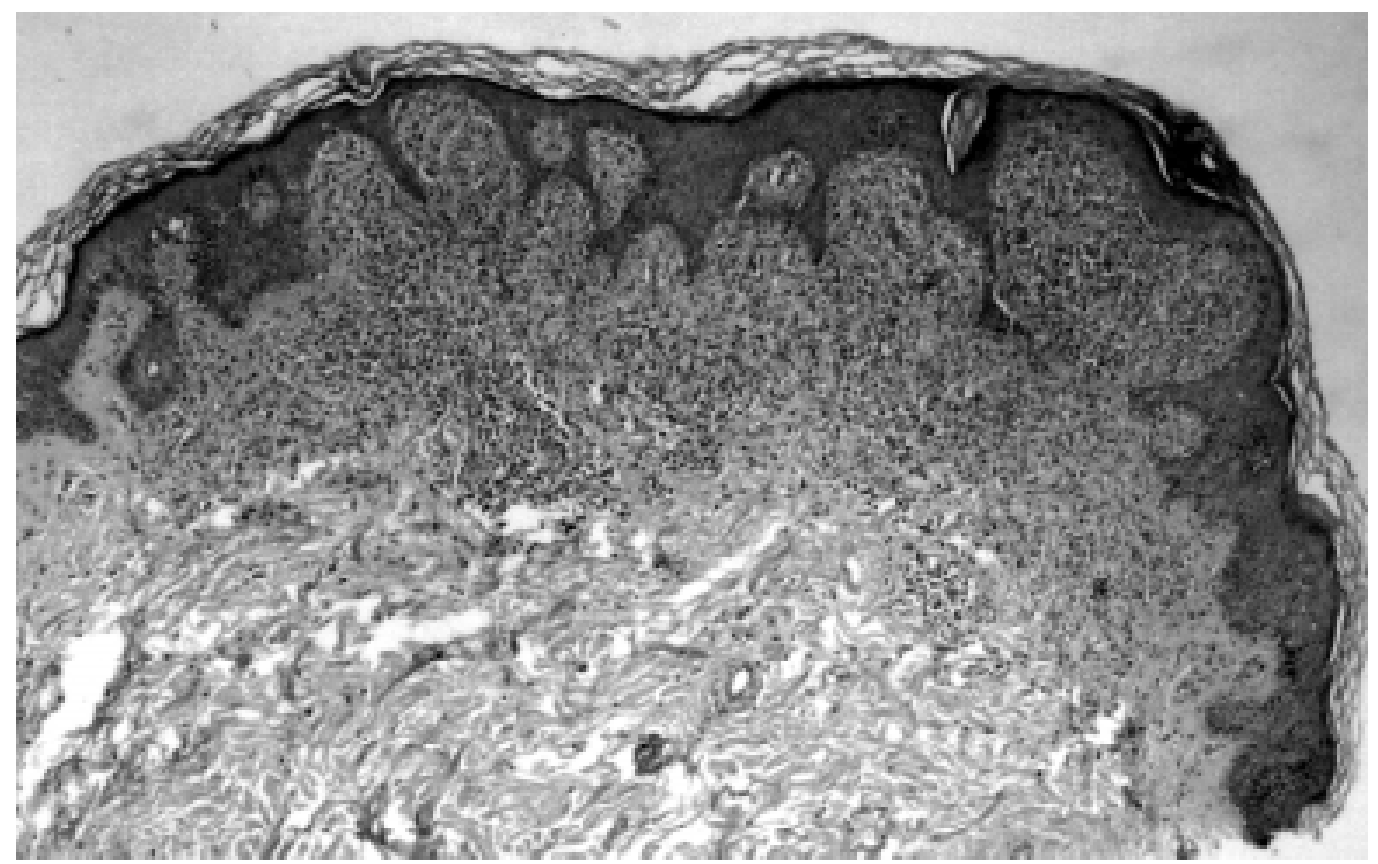

Figure 4. In greater magnification, parasitized macrophages in the papillary dermis. HE, A400.

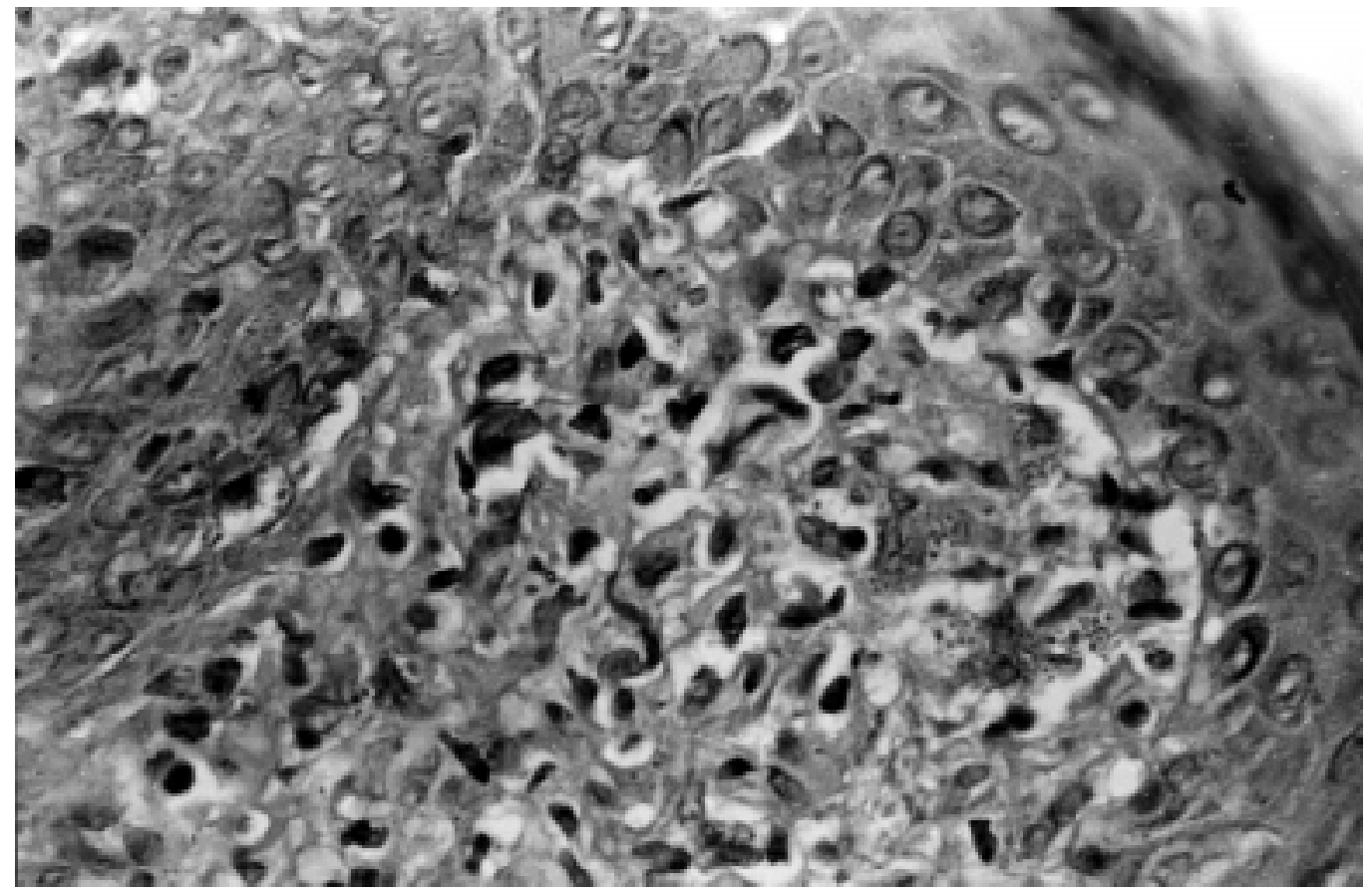


6. Montalban C., Martinez-Fernandez R., Callya J.L., et al. Visceral leishmaniasis (kala-azar) as an opportunistic infection in patients infected with the human immunodeficiency virus in Spain. Rev Inf Dis 1989; $11: 655-60$.

7. Sendino A., Barbado F.J., Mostaza J.M., et al. Visceral Leishmaniasis with Malabsorption Syndrome in a Patient with Acquired Immunodeficiency Syndrome. Am J Med 1990;89:673-5.

8. Viana G.M.C., Lewi D.S., Olzon P., et al. Leishmaniose Visceral e Síndrome da Imunodeficiência Adquirida (SIDA). Relato de um caso. Rev Inst Med Trop São Paulo 1994;36:384-6.

9. Ridolfo A.L., Gervasoni C., Antinori S., et al. Post-kalaazar dermal leishmaniasis during highly active antiretroviral therapy in an AIDS patient infected with Leishmania infantum. Brit Infect Soc 2000;40:199-202.

10. Cabrera R.A., Garcia C.G., Tello E.D., et al. Leishmaniasis cutánea post-kala-azar en un paciente con inmunodeficiencia adquirida. Actas Dermo-Sif. 1987;78:475-7.

11. Rios-Buceta L., Buezo G.F., Peñas P.F., et al. Post-kalaazar dermal leishmaniasis in a HIV-patient. Inter $\mathbf{J}$ Dermatol 1996;35:303-4.

12. Perrin C., Taillan B., Hofman P., et al. Atypical Cutaneous Histological Features of Visceral Leishmaniasis in Acquired Immunodeficiency Syndrome. Am J Dermatol 1995; $17: 145-50$.

13. Prata A., Domingues A. Leishmanióide dérmico. O Hospital 1955;50:93-113.

14. Badaró R., Jones T.C., Lorenço R., et al. A prospective study of visceral leishmaniasis in an endemic area of Brazil. J Infect Dis 1986; 154 :639-49.

15. Badaró R., Rocha H., Carvalho E.M., et al. Leishmania donovani: an opportunistic microbe associated with progressive disease in three immunocompromised patients. Lancet 1986;22:647-8.

16. Bittencourt A.L., Barral-Netto M. Leishmaniasis. In Doerr W., Seifert G., eds. Tropical Pathology. Berlin: SpringerVerlag, 1995.

17. Barral,A.; Costa,J.M.L.; Bittencourt,A.L.; et al. Polar and sub-polar diffuse cutaneous leishmaniasis in Brazil. Clinical and immunopathological aspects. Int. J. Dermatol. 1994;34:474-479. 